\title{
Clustering and Planning for Rescue Agent Simulation
}

\author{
Ahmed Abouraya, Dina Helal, Fadwa Sakr, Noha Khater, Salma Osama, \\ and Slim Abdennadher \\ German University in Cairo, Cairo, Egypt \\ \{dina.helal,slim.abdennadher\}@guc.edu.eg
}

\begin{abstract}
The paper describes the contribution of the GUC_ArtSapience team to the Rescue Agent Simulation competition in RoboCup in terms of the current research approach. The approach is divided into two parts: clustering and planning. Clustering is done through task allocation to divide the map among the agents. Planning is done after assigning the agents to parts of the map to determine how they should cooperate and coordinate together and how they should prioritize their tasks [2]. The agents can coordinate together using centers and communication if available or dynamically without the use of communication.
\end{abstract}

\section{Introduction}

Rescue planning and optimization is one of the emerging fields in Artificial Intelligence (AI) and Multi-Agent Systems. The RoboCup Rescue Agent Simulation provides an interesting test bench for many algorithms and techniques in this field. The simulation environment provides challenging problems that combine optimization (routing, planning, scheduling) and multi-agent systems (coordination, communication, noisy or missing communication) [3].

The Robotics and Multi-Agent Systems (RMAS) research group at the German University in Cairo (GUC) was established in September 2010. The goal of the research group is to study and develop AI algorithms to solve problems in robotics and simulation systems. These fields include computational intelligence, computer vision, multi-agent systems, and classical AI approaches.

The GUC_ArtSapience team made its third participation to the Rescue Agent Simulation in 2013 and won the first place. Our first participation (as RMAS ArtSapience) was in 2011 and the team ranked third in the final round. Our second participation was in 2012.

In this paper, we propose our approach which is divided into two parts: clustering and planning. Clustering is done using K-means to divide the map into small regions. The number of clusters is relative to the number of agents in the map. This is done in the preprocessing phase to set the initial positions of the agents in the map. This ensures that all regions in the map are covered by the three types of agents. As the simulation starts, each agent has a list of tasks to do which are prioritized. Agents communicate and coordinate together using communication channels and voice messages. 
This paper is organized as follows. Section 2 describes our clustering approach. Section 3 describes our planning technique regarding the agents' tasks and how they prioritize them. Section 4 describes our communication model. Finally, in Section 5 we give a summary of our results and performance in RoboCup 2013.

\section{Clustering}

In the rescue simulation environment, there is a lot of information that is obscure. For example, in disaster scenarios, the initial locations of fires, buried, injured civilians, and blockades are unknown. Moreover, tasks that are assigned to an agent do not specify where exactly this agent should carry out these tasks. Therefore, agents have no other choice but to traverse the whole map and search for their tasks. And since it is impractical for a single agent to cover the whole city map (all buildings and/or roads), our approach is to divide the map into smaller partitions.

Clustering is the method that we chose to divide the map. The clustering process is done in the preprocessing phase. Each type of agents cluster the map among themselves, so the maps is divided into clusters three times for Ambulance agents, Fire Brigades and Police agents. Ambulance agents and Fire Brigades clusters are groups of buildings while Police agents clusters are groups of roads close to each other. Each agent is assigned to a cluster, where it traverses all of the buildings/roads in it searching for events that require rescue actions. Figure 1 shows an example of Fire Brigade agents clustering the map in the preprocessing phase.

\section{$2.1 \quad$ C-means}

Initially, we used fuzzy c-means for clustering to generate overlapping clusters. Cmeans clustering is a method of clustering that allows one piece of data to belong to one, two or more clusters. The number of clusters was chosen to ensure that each cluster would have more than one agent. However, several tests showed that increasing the number of clusters to be equal to the number of agents produces better results. The problem with that was that increasing the number of clusters required extra computational time which exceeded the limited time allowed for preprocessing [6]. The advantages of using C-means clustering is overcoming the fact that there exist blockades all over the map which might prevent agents from reaching their targets. By increasing the number of agents heading for a specific target, the probability of this target being covered increases. However, time is wasted if two agents head for the same target.

\subsection{K-means ++}

$\mathrm{K}$-means++, as well as K-means clustering [5], aims to partition $\mathrm{n}$ observations into $\mathrm{k}$ clusters in which each observation belongs to the cluster with the nearest 


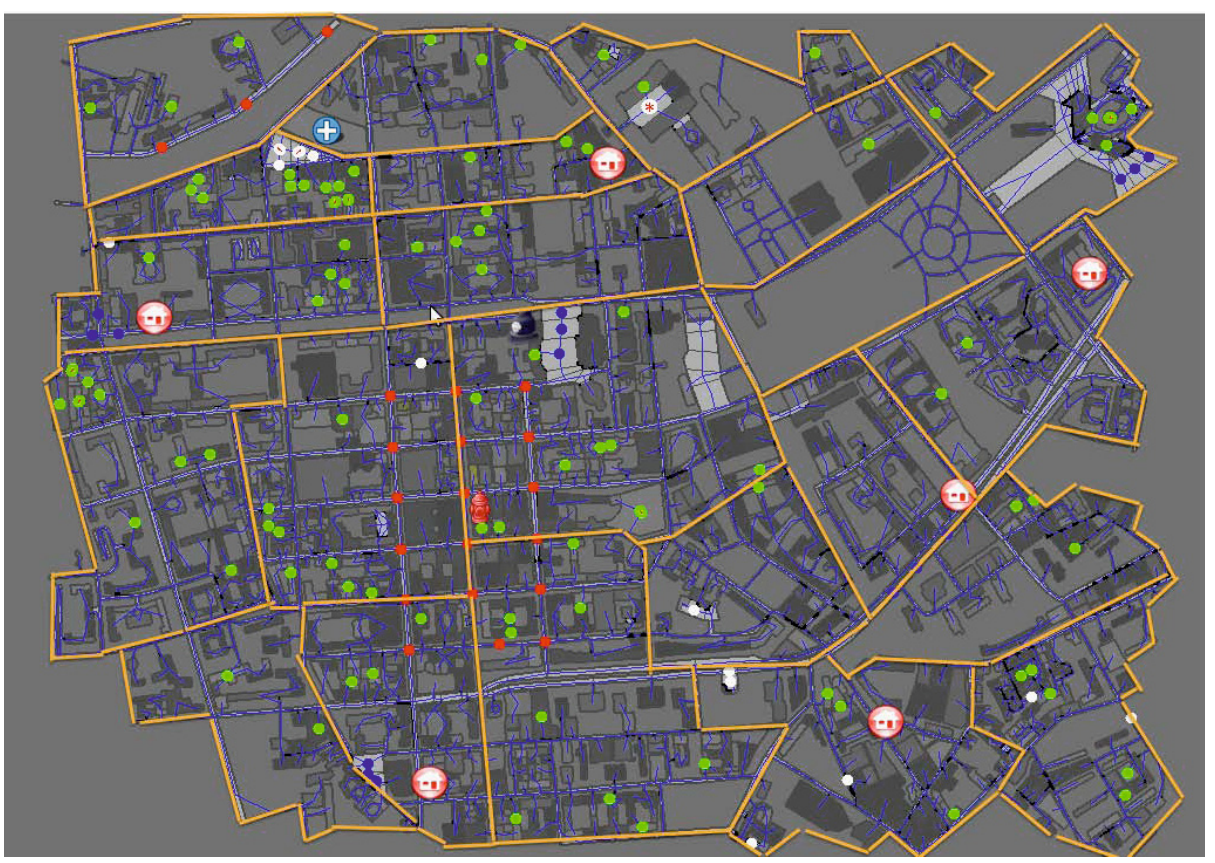

Fig. 1. Fire Brigade agents clustering the map

mean. To avoid sacrificing cluster quality, we chose to use K-means ++ clustering algorithm, which works similar to K-means, but uses a different heuristic for selecting the initial centroids which are selected from a uniform Gaussian distribution over the buildings in the map. K-means++ gives a much better start for the algorithm as well as guarantees a faster conversion to the optimal centroids. After dividing the map into partitions equal to the number of agents of a certain type in the map, each agent is assigned to the closest cluster to it.

Using K-means++ algorithm, we were able to overcome the time issue and we were able to compute clusters equal to the number of agents within the allowed time. Since the K-means++ algorithm is faster than the fuzzy C-means algorithm, we were able even to perform more computations during preprocessing such as calculating the shortest path distance between entities. In addition, through running the simulation on different maps, it was clear that there is a need for good strategies to find good starting values to reach the best possible solutions [4.

\section{Planning}

After assigning each agent to a certain region of map using the clustering technique, each agent has a set of tasks to perform as shown in figure 2, In this section we discuss the tasks of the Fire Brigades, Ambulance Team and Police Agents and how they are prioritized. Figure 


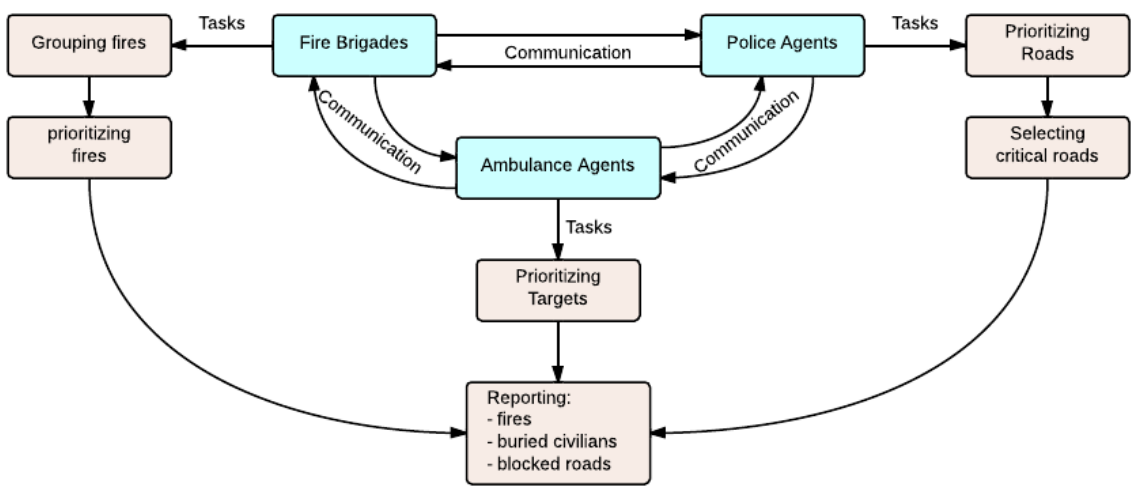

Fig. 2. Agents tasks after clustering

\subsection{Fire Brigades}

The main task of Fire Brigades is to extinguish fire in buildings. Thus, the Fire Brigades' targets in the map are buildings on fire. In the preprocessing phase, Kmeans ++ algorithm is used to partition the map into clusters which are groups of buildings close to each other on the map. Each cluster is then assigned to the nearest Fire Brigade agent. Each agent keeps searching for fires inside its own cluster. Once it finds one, it starts extinguishing the fire and reports it to the other agents using radio communication.

\subsubsection{Grouping Fires}

Sometimes a single agent can not handle a fire inside its cluster by itself. Therefore, other agents respond to the fire report by going to the fire location and help extinguishing the fire. A problem might arise if all agents responded to the fire report leaving unprotected parts in the map where another fire can start with no one to extinguish it. Therefore not all agents should respond to the fire report, only the closest ones head to the fire. This is handled by grouping fires together where close fires are considered to be belonging to one group with a center c and radius r. Agents are distributed among different groups of fire such that an agent is assigned to the closest group. Thus, the agents can handle more than one fire simultaneously.

\subsubsection{Prioritizing Fires}

Warm buildings are reported by Fire Brigade agents and considered to be on fire until their temperature is below a certain threshold. Extinguishing a building if it is warm is easier than extinguishing them if they are on fire. Extinguishing warm buildings helps in containing the fire because warm buildings are usually at the outer part of a fire group. Based on that, the highest priority is given to the 
warm buildings, then to buildings with lowest degree of fieryness (a parameter to measure the degree of fire in a building) then to buildings of the highest degree of fieryness. This way, the outer part of the fire group is targeted first which increases the possibility of containing the fire.

\subsection{Ambulance Teams}

The Ambulance team's main task is to rescue buried civilians and carry them to refuges so their target is buried civilians. Each Ambulance agent is assigned to a cluster which is a group of buildings like the Fire brigades. An ambulance agents searches for buried civilians withing its cluster then moves on to other clusters.

\subsubsection{Prioritizing Targets}

Each Ambulance agent keeps track of the buried civilians it came across but couldn't rescue because of blockades or fires. Accordingly, Ambulance agents divide their tasks into three groups. The highest priority is assigned to the currently seen buried civilians followed by the ones it previously passed by but could not reach followed by the reported targets seen by other agents.

A problem that Ambulance agents face is deciding how many Ambulance agents should rescue a certain civilian. As the number of agents increase the rescuing process takes less time, also as the burriedness of a civilian increases, more time is required to rescue him. To tackle this problem first, we tried to calculate and estimate death time for the buried civilians using their buriedness and damage parameters retrieved from the simulator. The buriedness parameter measures how much a civilian is buried under a building. When its value increases, this means the civilian is buried deeper. However, this was rather hard to do because of inaccurate simulator parameters. Based on that, we limited the number of agents rescuing one civilian to a certain number which is a function of the total number of Ambulance agents in the map and the burriedness of the civilian. This ensures that not all agents would try to save one civilian leaving other civilians to die.

Another problem they face is deciding which civilians need to be rescued first and which civilians should not be rescued as they might not make it to the refuge. This is calculated using a threshold where civilians with damage less than a certain threshold are left behind as they most probably will die before reaching the refuge. If the damage is higher than the threshold, higher priority is given to the most damaged civilians followed by the less damaged.

A decision the Ambulance agents need to make is deciding which agent should load the civilian if more than one agent were rescuing him. Only one agent can load a civilian so we let the agent with the lowest id load the civilian. This is based on the fact that it won't matter which agent loads the civilian and takes him to the refuge so it is just a systematic decision.

\subsection{Police Agents}

The last type of agents we are going to discuss is the Police agents. The Police agents' main task is to clear block road so that other agents can move freely 
in the map and perform their tasks. Each Police agent is assigned to a certain cluster which is a group of roads unlike the previous two agents which clustered the map in groups of buildings. Police agents divide their tasks into two parts which are prioritizing their targets and determining the critical blockades.

\subsubsection{Prioritizing Roads}

Police agents clear blocked roads as they move. Their highest priority is clearing roads leading to buildings on fire so that Fire Brigades can reach the buildings and extinguish the fires. The second priority is for clearing roads with agents stuck in them so that they can start performing their tasks. The third priority is clearing roads leading to refuges where Fire Brigades need to go and refill water and civilians need to go to e rescued. The fourth priority is for roads leading to buried civilians. Finally the lowest priority is for the rest of the blocked roads.

\subsubsection{Selecting Critical Roads}

Critical blockades are blockades which blocking any of the roads or buildings entrances. If the distance two blockades on the same road is smaller than the agent's diameter, then this blockade is considered to be blocking the road. If the blockade passes this first check, we project the blockade's shape on each of the open road edges. Then, we check whether each of the edges is passable or not. If an edge is not passable, then this blockade needs to be cleared. These checks are performed on all the blockades within the Police agent's clearing range and that are on the path to its current target. This saved a lot of time that used to be wasted on clearing non-critical blockades on the roads where the agents could pass right by them.

\subsection{Common Tasks}

The common task between all types of agents is reporting what they see. All agents report anything in their line of sight while executing their tasks. They report blocked roads to the Police agents, buried civilians to the Ambulance agents and fires Fire Brigade agents. Communication is done using radio or voice communication which will be discussed in details in the following section.

\section{Coordination and Communication}

A very important part of the rescuing process is the ability of agents to communicate with each other. Some scenarios allow the agents to subscribe to communication channels and send broadcast messages to each other. In other scenarios, there is no communication, only voice messages that can be send from one agent to its closest neighbor agents. We adapt our algorithm to handle both kinds of communication. Communication channels do not always send accurate message, sometimes there is noise that distorts the sent messages. 


\subsection{Communication Strategy and Approach}

In the scenarios with communication channels, the agents and centers subscribe to the communication channels according to available number of radio channels, maximum number of channels an agent can subscribe to, and the maximum number of channels a center can subscribe to [1].

The messages that are sent between the agents are prioritized according to their types. When agents send or receive messages, first they sort them in a priority queue based on the predetermined priorities. Such an approach allows the agents to utilize the resources and communicate efficiently, especially in the low communication or communication-less scenarios as the more important messages are given the higher priority.

In addition, a mapping technique that maps the IDs of various entities used in the communication process to IDs of smaller size has been devised. For instance, a road ID composed of a 6-digit number could be mapped to a unique ID which is a 2-digit number, thus reducing the message size by 4 bytes. Such an approach has proven to lead to better utilization of the bandwidth in case of radio channels and more information could be sent in a single voice message within the maximum allowed message size.

\subsection{Noise Handling}

Due to the heavy reliance of agents on communication, noise handling techniques are important to ensure that the messages are delivered and at the same to be able to make the communication process more efficient. In the communication channel, there are two types of noise: dropout noise and failure noise. Dropout noise is when the receiver receives a message without the content (i.e. content is lost), while failure noise is when the message disappears completely without notifying either the sender or the receiver. In order to overcome the noise, the messages are sent more than once. Each agent keeps track of the messages he/she sends per timestep. In the following timestep, the agent compares the messages heard with the messages that he/she sent through the channels during the previous timestep. During the comparison, the agent discards the duplicates and his/her own messages. The remaining messages which were sent by him/her and not received in the next timestep will be resent. This approach ensures that the cases of dropout or failure are handled without redundancy or resending of duplicates.

\subsection{Voice Messages}

Voice messages are important in scenarios with low communication or no communication at all. Each type of voice message has its own priority as explained in section 4.1. Furthermore, each voice message has its own time-to-live (ttl). The ttl basically limits the lifespan of the voice messages being propagated through the voice channels. Whenever a new voice message is created, it is set with the default ttl which is predetermined. Then as each agent receives a voice message, 
its ttl is decremented. If the $t t l$ is greater than 0 , then the agent rebroadcasts the message through the voice channel. And this process continues until the ttl of the voice message ultimately reaches 0 . Then the message is discarded and will no longer be propagated. Such an approach provides a means of broadcasting the voice messages across a broader range and overcoming the limitations of the absence or unreliability of the radio communication channels.

In addition, the agents ensure that the entire allowed maximum size of the voice message is being used through grouping the information which needs to be reported according to a specific priority and sending them in a single message. In this way, different information and data could be sent in a single voice message per timestep, thus leading to more efficiency.

\section{Results and Conclusion}

In 2013, the enhancements in the agents' performance and in the communication model paid off in the competition. After the first day, we ranked fourth out of 13 teams. After the second day, we ranked third out of 13 teams. In the semi-finals, we ranked fourth out of 8 teams which led us to the final round. In the final round, we outperformed the other 3 teams and came in the first place. There were 6 maps in the final round as seen in table 1. After the 5th map, we were in the second place, however the last map (Istanbul) put us in first place. The last map (Fig. 3) showed a huge difference in the performance between our team and the other three finalists (Fig. 4). Our map is the only one that got partially burnt while the other three maps are completely burnt by the end of the simulation which resulted in a huge difference in the score between us and the other teams.

In this work, we have described our approach that outperformed all other teams during the RoboCup 2013 finals. Our approach is divided into two parts: clustering and planning. Clustering is done using K-means to divide the map into small regions. The number of clusters is relative to the number of agents in the map. This is done in the preprocessing phase to set the initial positions of the agents in the map. This ensures that all regions in the map are covered by the three types of agents. As the simulation starts, each agent has a list of tasks to do which are prioritized. Agents communicate and coordinate together using communication channels and voice messages. Using our approach, our scores greatly improved in 2013 as we improved our strategy in prioritizing the agents' tasks as well as the coordination between them.

As a future work, it would be interesting to use the centers in communication. In addition, further improvements could be done in the agents' tasks such as predicting how the fire spreads so that the Fire Brigades can start extinguishing the buildings more likely to spread fire to their neighboring buildings. Also, civilians' health could be better estimated so that the Ambulance agents can decide whether the civilians can be saved or not which could eliminate the time wasted on rescuing civilians that die on their way to the refuge. 
Table 1. Rescue Simulation League 2013 Finals scores

\begin{tabular}{|l|l|l|l|l|l|l|}
\hline Map & Kobe4 & Eindhoven5 & Mexico3 & Berlin3 & Paris4 & Istanbul3 \\
\hline GUC_ArtSapience & 169.57 & 169.22 & 145.42 & 79.01 & 106.25 & 66.97 \\
\hline MRL & 170.51 & 165.92 & 156.16 & 66.14 & 84.64 & 12.90 \\
\hline Poseidon & 165.62 & 155.64 & 127.37 & 85.50 & 136.54 & 10.27 \\
\hline S.O.S. & 183.20 & 161.87 & 145.46 & 88.20 & 147.97 & 12.11 \\
\hline
\end{tabular}

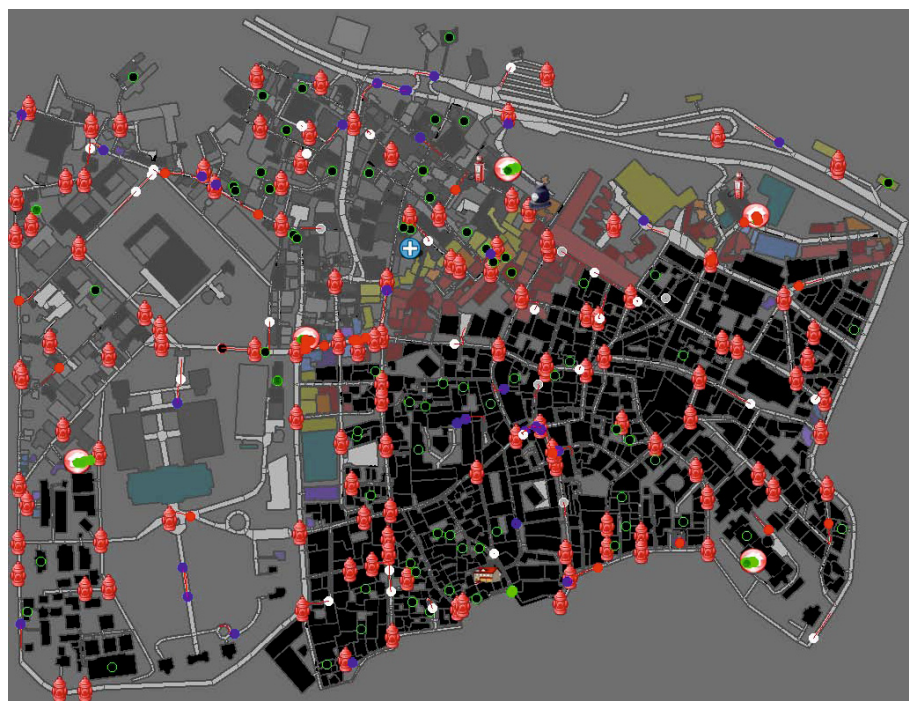

Fig. 3. GUC_ArtSapience Istanbul map in the final round
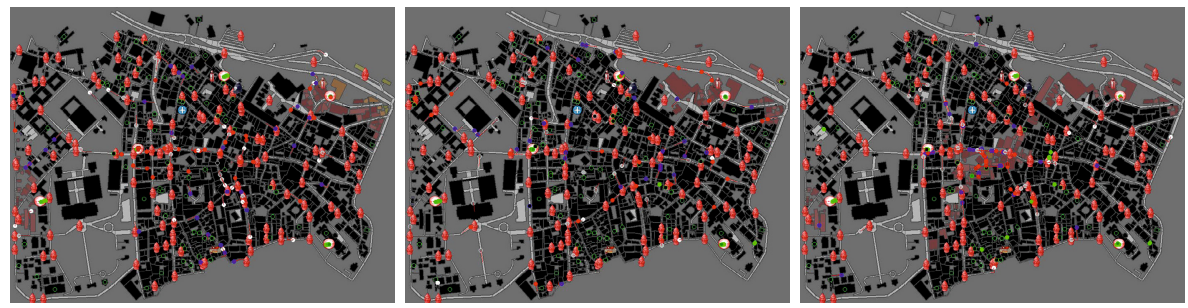

Fig. 4. Istanbul map of MRL, Poseidon, and S.O.S. in the final round

\section{References}

1. Guc artsapience team description paper (2012)

2. Multi-agent planning for the robocup rescue simulation: Applying clustering into task allocation and coordination. In: ICAART 2012 International Conference on Agents and Artificial Intelligence (2012)

3. Robocup rescue website, http://sourceforge.net/apps/mediawiki/roborescue/ 
4. Arthur, D., Vassilvitskii, S.: k-means++: The advantages of careful seeding. In: Proceedings of the Eighteenth Annual ACMSIAM Symposium on Discrete Algorithms, vol. 8(2006-13), pp. 1027-1035 (2007)

5. Kanungo, T., Mount, D.M., Netanyahu, N.S., Piatko, C.D., Silverman, R., Wu, A.Y.: An efficient k-means clustering algorithm: Analysis and implementation. IEEE Transactions on Pattern Analysis and Machine Intelligence 24, 881-892 (2002)

6. Winkler, R., Klawonn, F., Kruse, R.: Problems of fuzzy c-means clustering and similar algorithms with high dimensional data sets. In: Gaul, W.A., Geyer-Schulz, A., Schmidt-Thieme, L., Kunze, J. (eds.) Challenges at the Interface of Data Analysis, Computer Science, and Optimization. Studies in Classification, Data Analysis, and Knowledge Organization, pp. 79-87. Springer, Heidelberg (2012) 